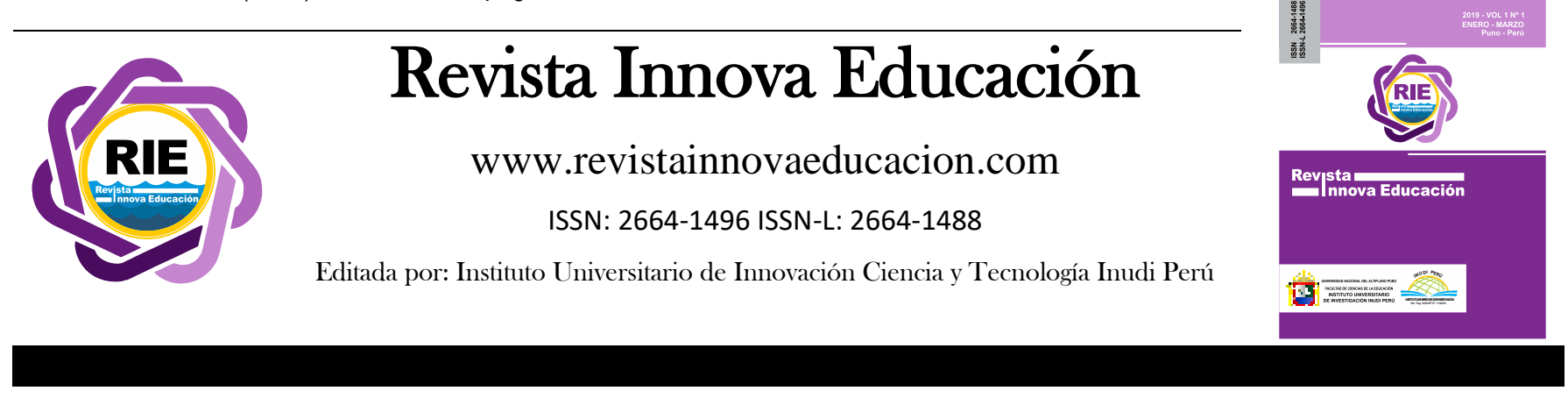

\title{
Acoso escolar y disrupción del aprendizaje en estudiantes de la secundaria de Chilpancingo, México
}

\author{
Bullying and learning disruption in high school students in Chilpancingo, Mexico \\ Yareli Reyes ${ }^{1}$ \\ Universidad Hipócrates, Acapulco de Juárez - Guerrero, México \\ (D) https://orcid.org/0000-0003-2727-2690 \\ John Acuña ${ }^{2}$ \\ Universidad Hipócrates, Acapulco de Juárez - Guerrero, México \\ (i) https://orcid.org/0000-0002-3686-7138
}

DOI: https://doi.org/10.35622/j.rie.2020.03.003

Recibido 15/05/2020/ Aceptado 25/07/2020 Publicado 29/07/2020

\begin{abstract}
ARTÍCULO ORIGINAL
PALABRAS CLAVE

Aprendizaje, acoso escolar, educación, escuela, violencia.

Ante la ola de violencia que padece el estado de Guerrero, México, resulta importante conocer los efectos de este fenómeno en el comportamiento y rendimiento académico de los estudiantes adolescentes. Asimismo, es necesario indagar las causas en las que se manifiestan las conductas violentas más habituales, así como postular alternativas locales e institucionales que mejoren la atención escolar de esta población altamente vulnerable ante el crimen organizado. Dicho esto, el presente trabajo tiene como propósito indagar sobre los elementos constitutivos que generan acoso escolar y disrupción de los aprendizajes en los estudiantes de la Secundaria Técnica $\mathrm{N}^{\circ} 185$ Hermenegildo Galeana de la ciudad de Chilpancingo. Para esto, se llevó a cabo un trabajo cualitativo de tipo etnográfico con la finalidad de explicar de una manera densa y detallada las relaciones de poder entre los estudiantes, así como las condiciones en las cuales se desarrolla el proceso de enseñanza-aprendizaje. De la misma manera, nos apoyamos de cuestionarios, encuestas y entrevistas para ahondar de mejor manera el propósito de esta investigación.
\end{abstract}

\section{KEYWORDS}

Learning, bullying, education, school, violence.
Given the wave of violence in the state of Guerrero, Mexico, it is important to know the effects of this phenomenon on the behavior and academic performance of adolescent students. Likewise, it is necessary to inquire the causes in which the most common violent behaviors manifest themselves, as well as to postulate local and institutional alternatives that improve school care for this highly vulnerable population in the face of organized crime. Having said this, the purpose of this work is to investigate the constitutive elements that generate bullying and disruption of learning in the students of Technical Secondary No. 185 Hermenegildo Galeana in the city of

\footnotetext{
${ }^{1}$ Correspondencia: yarita_aries21@hotmail.com

${ }^{2}$ Correspondencia: johnacuna@uhipocrates.edu
} 
Chilpancingo. For this, qualitative ethnographic work was carried out in order to explain in a dense and detailed way the power relations between students, as well as the conditions in which the teaching-learning process develops. In the same way, we rely on questionnaires, surveys and interviews to better deepen the purpose of this research.

\section{INTRODUCCIÓN}

El acoso escolar se ha convertido en uno de los fenómenos sociales que más ha afectado a la sociedad estudiantil, se presenta con mayor fuerza durante la adolescencia y se manifiesta de diferentes formas y modalidades como son: burlas, agresiones físicas, agresiones verbales y psicológicas. A pesar de las campañas de prevención de parte de la Secretaria de Educación Pública (SEP) y la información continua que circula en los diferentes medios de comunicación no se ha podido abatir su alta incidencia en los centros de instrucción pública. Al respecto, varios especialistas consideran que esta problemática merma el tejido social de las escuelas y, sobre todo, está obliterando el rendimiento intelectual, la autoestima y la estabilidad emocional de los estudiantes (Santoyo y Frías, 2014; Vega et. al., 2013; Muñoz, 2008; Ayala, 2015; Sánchez, 2013).

Este problema no está siendo considerado como uno de los elementos que dificultan el proceso de enseñanza y aprendizaje en la escuela. Al contrario, el acoso escolar es considerado como un aspecto externo a este proceso y que guarda relación con el carácter comportamental o individual del estudiante. Se desestima la idea de que el acoso escolar sea resultado de la manera cómo se reproduce la sociedad, es decir, como parte de una red de relaciones y cognitivas que están ligadas al tejido mismo de las estructuras sociales y económicas. Por ello, el interés de conocer los factores que influyen en la conducta de los jóvenes y sus efectos en el rendimiento escolar.

Cuando hablamos de acoso escolar (Bullying), nos referimos a todas aquellas conductas agresivas, intencionadas y repetitivas, con el fin de provocar un daño físico, psicológico o emocional en una o varias personas que, en su mayoría, no dan muestras de padecer agresión alguna (García et. al., 2015; Tresgallo, 2008; Gairín, 2013). Por lo general, los que ejercen la violencia son jóvenes que quieren demostrar superioridad frente a los demás y lo hacen por medio de constantes amenazas, insultos, golpes o empujones (Loredo-Abdalá, 2008; Armero, 2011). Los jóvenes que son víctimas (mujeres y hombres) de estas agresiones manifiestan comportamientos de aislamiento, viven intimidados y en constante angustia, padecen miedo todo el tiempo y suelen ocultar lo que están sufriendo. Prefieren callar y no denunciar el dolor que les causa la agresión; 
en algunas ocasiones, el acosado resuelve su problema a través de medidas o soluciones extremas como el suicidio.

Esta relación de dominación entre los adolescentes tiene que ser tomado en cuenta seriamente por las escuelas, porque se trata de una etapa compleja donde merecen mayor atención, sobre todo, en una sociedad violenta como la nuestra. En la actualidad, por ejemplo, con el uso de las tecnologías los adolescentes han empezado a viralizar las agresiones de sus compañeros, los exhiben en las redes sociales y hacen que la violencia sea vista o considerada como parte de una cultura. El acoso escolar debe ser pensado como un fenómeno que está destruyendo a la juventud. De este modo, podremos determinar las conductas sociales, psicológicas y/o psiquiátricas que influyen en el pensamiento de los adolescentes y que los orillan a tomar decisiones devastadoras como el hecho de quitarse la vida. Una vez reconocida estas determinaciones, las escuelas tomarán acciones concretas de prevención ante esta anomia, pero también generarán espacios de diálogo en la población estudiantil.

Durante el desarrollo humano los adolescentes manifiestan de diferente modo sus cambios psicológicos. En la escuela, les cuesta tener comunicación con los padres y maestros, prefieren ocultar ciertas emociones que las exteriorizan de distinto modo y entornos sociales. No obstante, esta situación es difícil de diagnosticar y atender en las instituciones educativas, sobre todo, públicas, porque no cuentan con un área de sicología especializada para brindar apoyo en caso de que exista un problema grave de violencia escolar.

Ante esto, creemos que la participación de otras dependencias gubernamentales es importante para coadyuvar y resolver el entramado que impide una sana socialidad entre los actores educativos, así como el logro académico esperado por las escuelas (Tresgallo, 2011; Satoyo et. al., 2014). Para ello, es indispensable conocer cómo se genera la violencia escolar y quiénes son sus agentes que la provocan, estos son: los compañeros, la familia, la escuela, la comunidad, entendida en su conjunto como defensora y transmisora de valores (Castro-Morales, 2011; Rodríguez et. al., 2009; Nocito, 2017).

\section{MÉTODO}

La presente investigación resalta por su carácter interpretativo y etnográfico (Rockwell, 1995; Rueda y Campos,1992; Bertely, 2000; Jociles, 1999). El trabajo se realizó en tres etapas: 
a) en la primera etapa se llevó a cabo una investigación analítica de las fuentes hemerográficas y bibliográficas con la finalidad de conocer el debate en torno al acoso escolar o bullying. Esto nos permitió construir un campo de conocimiento teórico sustantivo para que finalmente podamos interpretar los factores que delimitan las conductas de violencia en la Secundaria Técnica $N^{\circ} 185$ de Chilpancingo (Sautu et al. 2005).

b) en la segunda etapa, se puso en marcha un trabajo de campo que permitió realizar una descripción densa y minuciosa con base en la observación no participativa de los actores educativos, las víctimas y agresores estudiantiles (Geertz, 1986; Pérez, 2012; Guber, 2001; Bertely, 2000). En esta fase se realizaron 7 entrevistas abiertas y conversaciones en grupo con los estudiantes. Asimismo, se trabajó con 10 estudiantes identificados como posibles agresores escolares. Para saber más al respecto, se realizó una pequeña encuesta con el propósito de indagar sobre los factores disruptivos que condicionaban el comportamiento y el aprendizaje de los estudiantes.

c) en la tercera etapa se aplicó el Cuestionario de clima de aula y de centro para alumnos de Fernández et. al. (2002). La muestra fue no probabilística, se trabajó con 104 estudiantes. Estos estaban agrupados en el primer, segundo y tercer grado respectivamente. De estos estudiantes se destaca que 56 eran hombres y 48 eran mujeres. Las unidades de análisis de este estudio fueron los estudiantes. Los criterios de inclusión los alumnos inscritos en el año escolar vigente y quienes aceptaron de manera voluntaria ser incluidos en el estudio. Los criterios de exclusión fueron aquellos estudiantes que no aceptaban ser incluidos en el estudio y aquellos que tenían alguna discapacidad o problema físico.

\section{RESULTADOS Y DISCUSIONES}

\section{a) En torno al acoso escolar}

El acoso escolar es una forma de violencia que se realiza durante un tiempo determinado por parte de uno o varios estudiantes y tiene la intención de hacer daño físico o psicológico a uno o varios estudiantes (Enríquez y Garzón, 2015; Pedreira et. al., 2011). Cabe indicar que el acoso escolar incluye tres aspectos que se deben de resaltar o tomar en cuenta para su entendimiento: a) la amenaza es un comportamiento agresivo que implica acciones no deseadas; b) la provocación reafirma un patrón de comportamiento que se repite una y otra vez; y, c) la amenaza implica un desequilibrio de poder o fuerza que se ejerce sobre la víctima. De acuerdo con esto, existen varios tipos de comportamiento antisocial y estos son: a) disrupción en el aula; b) conflictos entre 
profesor y alumno, c) maltrato entre estudiantes; d) vandalismo; e) agresiones o extorsiones; f) acoso sexual (Rodríguez et. al., 2011, p.2).

Durante estos últimos años la falta de convivencia y la indisciplina en la escuela se han convertido en problemas que aquejan tanto a los profesores como a los responsables de la administración educativa. Esta preocupación surge por la frecuencia de casos de acoso escolar que en definitiva rompen con la buena armonía y convivencia de los centros educativos. Sin duda esto se debe a la dificultad de encontrar soluciones idóneas y eficaces para superar el conflicto entre los estudiantes. Nadie contradice que la convivencia y el respeto hacia las personas sean consideradas como aspectos necesarios para conseguir una enseñanza de calidad. Sin embargo, la violencia, la indisciplina y el descontrol en los centros educativos van en aumento en la sociedad contemporánea (García et. al., 2001).

Este hecho social no surge como un fenómeno propio de la escuela, ni se puede ser considerado como un aspecto aislado del cambio de las relaciones sociales y familiares que se da en el mundo. Quiere decir que las transformaciones sociales impactan directamente en los tejidos institucionales como son la escuela, la familia, los sistemas políticos locales, etcétera. De otro lado, dentro de los valores y actitudes sociales de la educación se encuentra el respeto a las reglas de convivencia como propósitos de un sistema educativo más democrático. Esto ayuda a entender que hay un orden moral en una sociedad donde, además, se puede desarrollar el autocontrol y la autonomía escolar. A esto se añade la idea de que la convivencia, el respeto y la disciplina escolar son elementos necesarios para lograr los fines y objetivos de la educación. De acuerdo con esto, podemos creer que todo estudiante necesita de reglas de convivencia y de respeto hacia los demás como:

1) El respeto mutuo le da un sentimiento de seguridad al ver hasta dónde puede ir y lo que debe y no debe hacer. 2) Al vivir de acuerdo con ciertas normas, el alumno es capaz de evitar sentimientos frecuentes de culpa que le asustan. 3) Ayuda a desarrollar su conciencia, la "voz interiorizada" que le guía a elegir por sí mismo. 4) Al cumplir las normas de respeto descubre que los demás le aceptan formando y desarrollando su socialización. 5) Aprende a comportarse de manera socialmente aprobada y con el tiempo a tener una sólida autodisciplina y autocontrol. 6) Le sirve de motivación para reforzar su yo y llevar a cabo lo que se espera de él (García, p. 165).

El respecto entre los individuos o el clima de relaciones humanas que predomina en las escuelas es una de las variables que más influye en el aprendizaje de los estudiantes (Collino, 
2017). Éste suele ser reflejo de las capacidades y de la propia gestión de control del centro escolar. Quiere decir que una escuela con buen clima escolar es una organización académica donde existe colaboración entre directivos, profesores, estudiantes y padres de familia. La eficacia y la mejora escolar son variables de gran importancia que ayudan a consolidar los objetivos del currículo escolar, independientemente del contexto en el que se encuentre el alumno.

Se trae a colación esta discusión, porque el acoso escolar representa un fenómeno específico de comportamientos agresivos repetidos en los que existe una diferencia sistemática de poder entre el victimario y la víctima. Esto puede involucrar acciones físicas, verbales, de exclusión social que afectan al individuo o a la comunidad (Rodríguez et. al., 2012; Blanchard et. al., 2007; Carnety et. al., 2001). De hecho, se ha señalado que es difícil medir el bullying puesto que se debe detectar el nivel de agresión, la intención del agresor, así como el ejercicio de poder entre los estudiantes (Vega-Cauich, 2018; Albores-Gallo et. al., 2011). Dada la naturaleza del bullying creemos que ésta afecta los resultados académicos y agrieta el clima escolar de una manera negativa. Se ha comprobado que en las escuelas donde prevalece el acoso escolar de una manera constante, existe un deterioro del capital social y cultural enorme. Los estudiantes no logran buenos resultados académicos y, por lo general, no culminan o desertan de las escuelas. Esto se debe a la falta de habilidades o gestión escolar para solucionar los conflictos de una manera estratégica y académica (Hamodi y Jiménez, 2018; Jankue, 2016).

La presencia de robos y agresiones de todo tipo son situaciones violentas que tienen lugar en las escuelas. Una buena convivencia entre estudiantes, profesores y directivos se relaciona positivamente con el logro académico, además, señala que las buenas relaciones entre distintas culturas favorecen el aprendizaje de los estudiantes (UNESCO, 2007). Pero, para eso es importante el papel que juega el profesor en la construcción de una escuela dialógica y menos violenta. Una relación fluida entre el alumno y el profesor tendría una alta correlación con buenos resultados académicos, en otras palabras, un buen clima escolar actúa siempre en favor de los agentes educativos.

En ciertas ocasiones el centro escolar puede llegar a convertirse en un espacio no propicio para el aprendizaje o la convivencia social. Esto se debe en gran medida al grado de violencia que existe en la escuela y a las condiciones para que el estudiante se integre al grupo. Es decir, la violencia escolar no necesariamente se realiza a partir de la relación compleja entre los estudiantes, sino también entre todos los agentes educativos como el docente, quien al igual que los que se 
encargar de gestionar o administrar la escuela no logran generar condiciones sociales óptimas para el aprendizaje.

\section{b) Disciplina e indisciplina}

No existe una definición única del término disciplina, puesto que ésta depende de la visión del profesor y de la filosofía institucional. No obstante, la consideramos a ésta como un aspecto inherente al desarrollo de la educación y que contiene un carácter positivo o negativo en el salón. Moreno et. at. (2007) sostienen que un acercamiento positivo hace referencia a toda forma de comportamiento que logra los objetivos programáticos o metas institucionales, mientras que una indisciplina negativa hace referencia a la ausencia de un diálogo entre los estudiantes y/o docentes. Esta forma de comportamiento necesariamente conlleva a un rechazo rotundo de parte del centro educativo e incluso implica un castigo sobre los sujetos que hacen uso del poder sobre el otro.

Para impedir esta sanción es necesario que los gestores educativos se organicen e impongan una disciplina con la finalidad no sólo de lograr las metas indicadas desde el inicio del ciclo escolar, sino también para incorporar en la sociedad a ciudadanos responsables y con valores (Vásquez, 2010; Sáenz-López, 1997; Maquilón, 2011). Cuando se hace referencia al control o vigilancia del estudiante se habla del reconocimiento de una variación de conductas que diagnostica el profesor y afectan el desarrollo de la clase. Esta etapa es de gran importancia, porque de esto depende la neutralización de comportamientos disruptivos y desestabilizadores de las relaciones académicas. De acuerdo con esto, Gómez y da Resurrección (2017, p. 279) señalan que:

El término disrupción se refiere a las conductas que llevan a cabo aquellos alumnos dentro de las clases que pueden buscar diferentes objetivos como llamar la atención, reclamar su deficiente historia académica, pero que tienen como consecuencia que el profesorado no pueda llevar a cabo de manera adecuada su tarea profesional de enseñanza, impidiéndole que pueda hacer la explicación de los temas, realizar las actividades oportunas o aplicar las evaluaciones oportunas que considere necesarias.

La conducta disruptiva es generada por un cierto tipo de factor que interviene en la forma de pensar del estudiante y alteran la "normalidad" del centro escolar (Orrego, 2014). Las soluciones a este tipo de disrupciones pasan necesariamente por la realización de una investigación que identifique las probables causas que ocasionan malestar en el estudiante. A groso modo, estas 
causas o factores pueden ser: a) Factores sociodemográficos, asociados al género del alumno, a las condiciones de pobreza, la edad, la lengua o la etnia; b) Factores educativos, asociados a la burocracia educativa, los planes y programas, la filosofía institucional y los estilos de enseñanzaaprendizaje; c) Factores sociales, asociados al lugar de origen, la situación laboral de los padres; y, d) Factores familiares, asociado al conflicto de los padres, el maltrato o la violencia física (Garbanzo, 2007).

Es posible que estos factores contribuyan al resquebrajamiento de la convivencia escolar y probablemente sea necesario intervenir desde distintas instancias públicas en el sistema educativo (Escudero, 2005; Mena et. al., 2010). De acuerdo con esto, la conducta disruptiva influye negativamente en el ejercicio docente y, también, agrava las relaciones sociales y educativas en la escuela (Ovalles, 2017, p.32). Ante esto, los docentes deben detectar cómo afectan las conductas disruptivas en el desarrollo de la clase y para ello es necesario considerar como una tarea sustantiva una radiografía de los factores disruptivos que condicionan el quehacer del estudiante. Esta tarea debe de ir de la mano con las instancias internas de la escuela que se encargan de llevar a cabo una atención (psicológica) del estudiante.

Por lo general, esta acción se realiza con el firme propósito de reducir actos o conductas disruptivas que vayan en detrimento de los intereses de la institución. Además, el diagnóstico que se vaya a realizar podría prevenir el fracaso y abandono escolar. La disrupción es un problema que impacta en el aprendizaje, la convivencia y el abandono escolar, además entorpece las acciones que se llevan a cabo en el aula e implica perjuicios tanto para el educando como para el docente. Estos factores obligan al docente a dedicar gran parte de su tiempo a la atención de los estudiantes con problemas familiares, económicos o socioculturales.

\section{c) La violencia escolar como trabajo de campo}

El día 7 de febrero de 2020 se realizó una encuesta a tres grupos de la Secundaria Técnica $\mathrm{N}^{\circ} 185$ Hermenegildo Galeana. Esta actividad se llevó a cabo con la finalidad de recabar datos que ayudaran a fortaleces la presente investigación. En dicho centro educativo se tomó como muestra a un grupo de primer grado, uno de segundo y uno de tercero con un total de 104 alumnos, de los cuales 56 pertenecían al género masculino y 48 al género femenino. Esta actividad arrojó como resultado las siguientes gráficas que esbozan el trabajo realizado antes de que se produjera el cierre de las escuelas, producto de la pandemia conocido como COVID 19 que padece el mundo. Cabe indicar que al momento de aplicar la encuesta nos dimos cuenta que muchos de los alumnos desconocían de algunos términos considerados por la encuesta (Fernández et. al., 2002). La 
complejidad de este instrumento nos obligó a explicar de una manera didáctica algunos temas como disrupción, acoso o violencia escolar, conducta y aprovechamiento escolar con varios grupos pequeños de 5 o 7 personas. El resultado de esta actividad culminó en la aplicación de la encuesta tal como se puede observar.

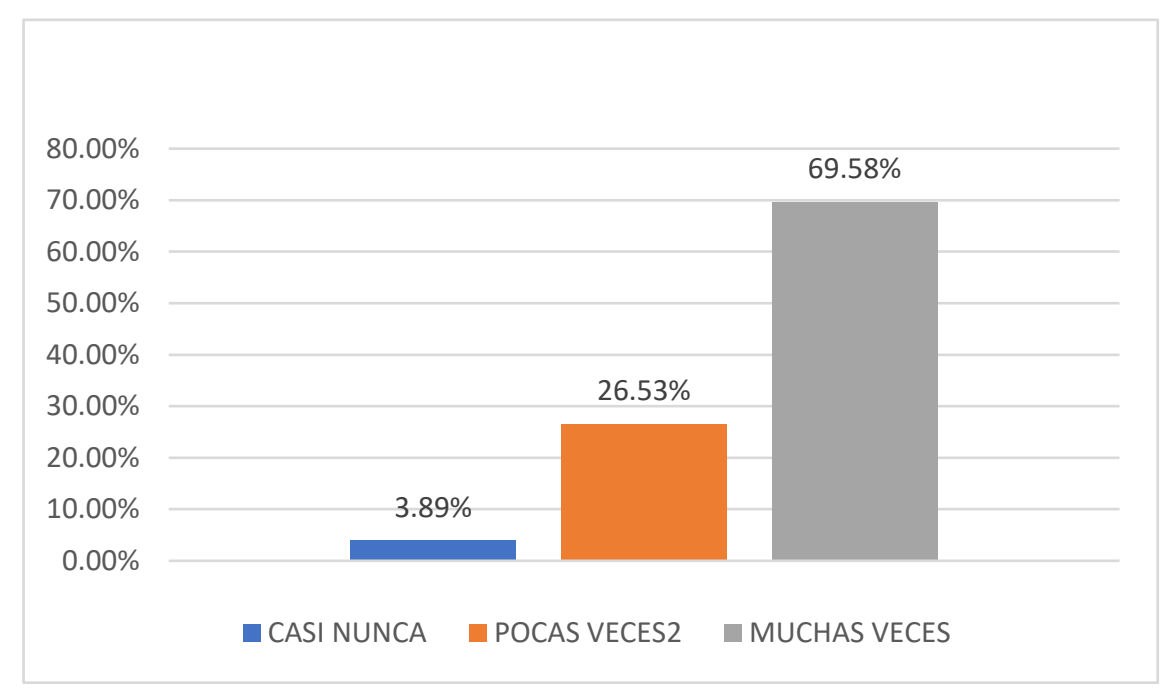

Figura 1: Mujeres estudiantes que han padecido alguna forma de acaso escolar.

De acuerdo con el gráfico precedente, Figura 1, cuando se les preguntaba a las mujeres si habían sido agredidas o habían sufrido en algún momento una forma de acoso o violencia escolar de parte de sus compañeros y compañeros, el $69.58 \%$ de las 48 mujeres señalaba que había padecido en muchas o reiteradas ocasiones un tipo de violencia. Durante el trabajo de campo realizado, nos dimos cuenta que el acoso escolar en las mujeres estaba asociado a los factores disruptivos o comportamentales proveniente de los agresores, quienes tenían problemas familiares, económicos y sociales. Esto fue un aspecto importante para nuestro trabajo, porque nos permitió realizar una encuesta o sondeo a un número determinado de estudiantes, diez en realidad, que cometían, según los estudiantes, actos de acoso escolar. El acoso era cuestionado por las mujeres, una estudiante nos dijo lo siguiente:

Las personas que agreden a las compañeras tiene problemas con sus papás o, algunos, vienen con mucho sueño o trabajan, no sé. Solo vienen a la escuela a molestar. Yo he visto a mucha de mis compañeras que se sienten tristes, las veo decaídas y, a veces, tienen poca autoestima. Los profesores han visto estos casos en clases, pero no les prestan mucha atención. Sus ocupaciones son diversas que hacen otras cosas (Entrevista A, febrero 2020). 
La impugnación de nuestra entrevistada al acoso escolar provocado por sus compañeros tiene muchas significaciones. Nos parece que su testimonio resalta en alguna medida aquello que no se logra discutir en la escuela y esto tiene que ver con la violencia y la lucha de poder que existe entre los estudiantes acaso los docentes. Cuando la entrevistada A indicaba que el estudiante "tiene problemas con sus papás", nos dimos cuenta que subiste un comportamiento disruptivo asociado a un problema familiar o incluso cuando señalaba que su compañero acude la escuela con "sueño y trabaja", evidentemente hacía referencia a un carácter disruptivo de tipo económico.

Como se ha indicado arriba, la disrupción en los jóvenes se da con la intención de llamar la atención y provocar de algún modo una acción incómoda en la escuela. Evidentemente, esto implica una respuesta inmediata de parte del docente y, desde luego, una pronta desatención de sus obligaciones académicas. Es decir, el proceso de enseñanza-aprendizaje se ve obturada por este tipo de problemáticas que son recurrentes en escuelas donde persiste acoso escolar. Desde luego, la resolución o respuesta del profesional de la enseñanza no viene acompañada de una plataforma institucional que de asistencia a este tipo de acciones o comportamientos disruptivos de parte de los estudiantes. Hemos notado que, si bien existe un centro de atención sicológica en la Secundaria Técnica, no existe un seguimiento al estudiantado como tal. Es más, por lo general, el docente se ve ocupado en diversas tareas que le impiden prestar atención a comportamientos complejos. De otro lado, es necesario indicar que en la secundaria este problema no sólo afecta a las mujeres, también los varones han padecido de violencia escolar.

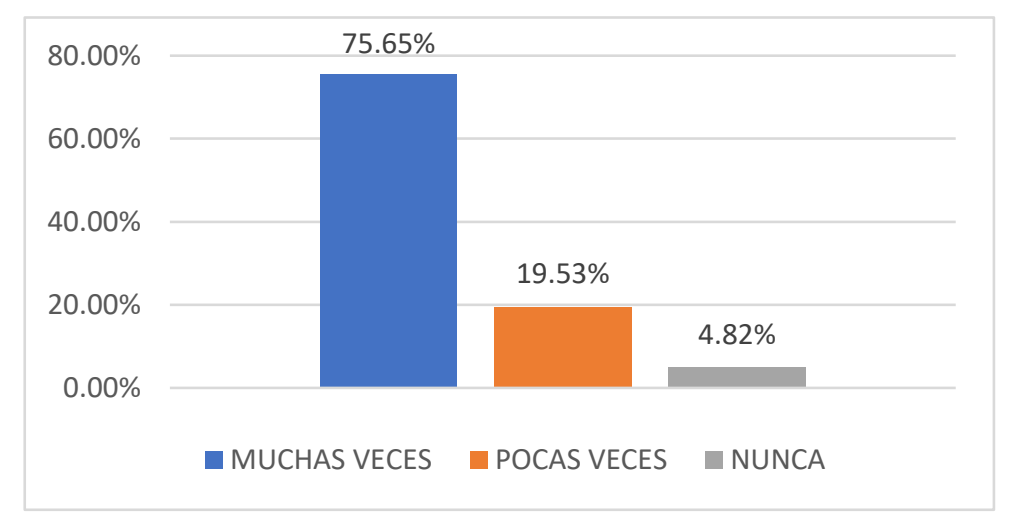

Figura 2: Hombres estudiantes que han padecido alguna forma de acaso escolar.

En el cuadro de arriba, Figura 2, se puede ver que el $75.65 \%$ de 56 hombres ha padecido en reiteradas o muchas veces acoso escolar. A diferencia de las mujeres, los hombres parecieran sufrir más acoso que éstas, pero lo cierto es que las relaciones entre los varones son mucho más agresivas. Este dato no implica que este grupo de hombres no ejerza poder o violencia alguna, al 
contrario, son los hombres lo más propensos a sufrir y ejercer alguna forma de violencia escolar. En el trabajo de campo, hubo un testimonio que nos llamó poderosamente la atención, pues no sólo se podía identificar la pugna entre los estudiantes, sino también la reafirmación de una problemática presente en el país, nos referimos al machismo.

El acoso se da más entre varones, las mujeres también participan, pero no es mucho o no se nota, pero si lo hacen. Entre los varones hacemos y decimos cosas. En muchas ocasiones tenemos que aguantarnos, porque se burlarían más. Se reirían o nos molestarían las mujeres [...] En una ocasión vi que a un compañero su puso a llorar y pensaban que era bisexual, por eso le hacían bullying. Le decían que es joto, que es puñal (Entrevista B, febrero 2020).

Cuando el entrevistado B indicaba que sus compañeros "se burlaban” de él (posiblemente) y lo clasificaban, lo que estaban haciendo sus compañeros era reafirmar una relación escolar que giraba en torno a la masculinidad. Norma Fuller (2001), por ejemplo, menciona que las masculinidades se definen también a partir de la coerción sobre el otro y, desde luego, sobre una figura social reconocida por lo general como hombre. En el caso que nos interesa, señalamos que esta forma de discriminación no puede ser entendida sin considerar las contradicciones de la anatomía de la sociedad, en este caso, la guerrerense o mexicana. La burla sobre la debilidad o la feminidad es un aspecto que se reproduce de manera constante en la escuela. Pero, también da cuenta de una sociedad falocéntrica que legitima la violencia. Cuando se hizo una entrevista abierta a un estudiante, él señaló lo siguiente:

Entrevistador: ¿Conoces a algún compañero que se haya sentido mal después de recibir un insulto? Sí (estudiante). Entrevistador: ¿Cómo lo notaste? Triste, se pone a llorar, le hacemos burlas por que llora (estudiante). Entrevistador: ¿Qué le dicen cuando llora? Que es una niña, cosas feas, que es gay y esas cosas (estudiante). ¿Ese niño actualmente viene a esta escuela? No, ya se fue...creo que tiene vergüenza, ya no estudia (Entrevista C, febrero 2020).

Hemos notado que la violencia o acoso escolar que se da entre los estudiantes no se logra percibir o no está siendo considerado como un verdadero problema que afecta el logro académico de los estudiantes. Muchos de ellos, vale decir, las víctimas prefieren callar y no contar de los maltratos que sufren. Esto se debe al miedo y la vergüenza que genera el acoso en la escuela. El testimonio siguiente afirma lo dicho: 
Una vez grabaron a una compañera...Pues, estaba en los baños y un niño se metió y la grabaron. La niña ya no viene a esta escuela, la cambiaron, se fue lejos. El niño también se fue, no sé a dónde (Entrevista D, marzo de 2020).

Otro entrevistado, nos señala algo similar.

He visto a compañeros en una situación vergonzosa. Algunos han sido grabados y sus fotos o videos se han publicado en las redes sociales. También he visto a mis compañeros jugar muy feo...Una vez a un compañero lo estaban empujando, haciendo feo y lo estaban grabando. El otro no podía defenderse, lo atacaban entre varios. No hizo nada. Solo se estaba riendo, pero se veía que quería llorar (Entrevista E, febrero de 2020).

La violencia entre los jóvenes puede salirse de descontrol. En la secundaria observamos que los jóvenes actúan sin medir las consecuencias. No existe un proyecto institucional que se ocupe de prevenir estos actos de violencia entre los estudiantes. Hemos notado que los docentes no desean reconocer que este problema no guarda relación con la edad de los adolescentes. Se trata de un asunto muy preocupante. La omisión a estos casos podría generar una situación muy complicada de controlar para la secundaria. En alguna ocasión, cuando se les preguntaba a los estudiantes si los profesores hacían algo al respecto, la respuesta era que no, es más, alguno de ellos ejercía violencia entre los estudiantes o las generaba.

No todos los profesores hacen eso...molestan a los estudiantes. Pero, un profesor sí. Una amiga de mi salón tiene el pelo corto y luego [él] le anda pegando cosas, le puso un apodo, pero no me acuerdo como era, la niña le dijo al profe que no se lo dijera (Entrevista F, marzo 2020).

Como ya se ha indicado, el acoso escolar no necesariamente es provocado por el estudiante, sino también por otros agentes educativos. Los docentes sin querer agredir al estudiante pueden estar normalizando las relaciones complejas entre los estudiantes. Ante esto, la exigencia de ayuda de parte de los agredidos se manifiesta a veces de una manera corporal. Nos referimos al cutting, mutilación del cuerpo, se trata de un fenómeno que ha ido creciendo y pasa desapercibido en algunos centros escolares. Al respecto, alguien nos narró lo siguiente:

Una vez vi a una compañera que se lastimaba así misma. Iba en mi salón y siempre parecía estar tranquila, pero había ocasiones que reaccionaba de mala manera. Eso fue en primero, no sé si lo siga haciendo, como no vive con sus papás, vive con sus 
abuelitos y eso le afecta mucho. Se empezó a cortar y así. Le he visto cicatrices...Sí, en sus manos. Yo noto que se siente sola, tiene mucha tristeza, porque la abandonaron con sus abuelos (Entrevista G, marzo 2020).

En la Técnica $\mathrm{N}^{\circ} 185$ hemos logrado detectar que la mayoría de estudiantes tienen comportamientos que están relacionados a cierto tipo de problemas o factores disruptivos económicos, sociales y familiares. En ese sentido, se hizo un pequeño sondeo a diez estudiantes que eran considerados por sus compañeros como agresores. La mayoría de ellos afrontaba problemas económicos, la otra parte tenían problemas sociales y muy pocos problemas familiares. Lo que nos llama la atención de este aspecto es que los factores disruptivos sí inciden sobre el rendimiento en clase de los estudiantes.

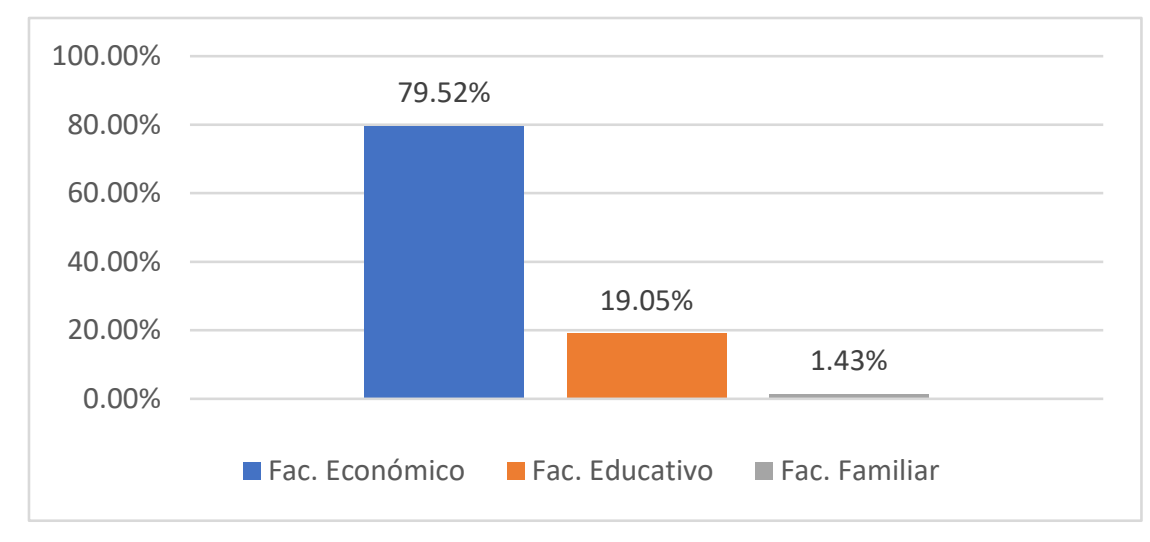

Figura 3: Factores disruptivos que afectan el bajo rendimiento académico.

Los problemas económicos a los que se referían los estudiantes tenían que ver con el trabajo que realizaban los padres. Gran parte de los estudiantes señalaba que los papás se encontraban todo el día fuera de casa y que sólo los veían unas horas al día. Asimismo, otro sector señalaba que, debido a la falta de dinero en sus hogares, tenían que ayudar para solventar los gastos. Esto implicaba el abandono escolar por temporadas cortas. Otro factor presente en la encuesta guardaba relación con el aprendizaje o factor educativo. Los estudiantes que pertenecían a este grupo indicaban que no lograban captar lo que aprendían, porque las clases no eran amenas o, finalmente, los estilos de enseñanza de parte de los docentes no llamaban su atención. Asimismo, se logró identificar en este factor social que la violencia escolar también era consentida y, en algunas ocasiones, reproducida por los propios docentes. De otro lado, muy pocos estudiantes de este grupo culpaban a la falta de atención o aprendizaje de sus estudios a la administración o atención escolar. Por último, el otro sector que aparece es el de la familia, no obstante, el problema familiar o el problema de los padres o el maltrato que sufren los adolescentes es muy bajo respecto a los factores 
sociales o académicos. Habría que desarrollar un trabajo más complejo para conocer a ciencia cierta si éste es un factor que impacta en el comportamiento de los estudiantes.

\section{CONCLUSIONES}

Consideramos que el trabajo merece una mayor atención. Lamentablemente, la pandemia, COVID19, impidió un mayor desarrollo del problema analizado. Sin embargo, en términos generales, podemos decir que el acoso escolar continúa siendo considerado como un problema inherente a la dinámica escolar. Ésta no es pensada como una de las causantes que impiden el disloque de los aprendizajes y rendimientos académicos. No obstante, creemos que el acoso escolar no tiene que ver con una problemática interna, sino con su articulación con los problemas sociales que arrastra la sociedad (guerrerense). La violencia en todas sus formas son manifestaciones culturales que se reproducen en todos los ámbitos culturales y políticos. La escuela no es una entidad separada o que se encuentre aparatada del comportamiento mismo de la anatomía de la sociedad. En la investigación realizada, nos hemos dado cuenta que los problemas de acoso escolar generados por el agresor tienen una explicación que está enraizada por la dinámica de la vida cotidiana. En otras palabras, los factores de disrupción de las relaciones escolares y académicas, por qué no comportamentales, como la carencia económica, la discriminación y el problema familiar inciden directamente en el estudiante. Se ha notado en este trabajo que los estudiantes que provienen de familias con pocas oportunidades económicas son las que realizan actos de agresión física o verbal contra sus compañeros; son muy pocos los que tienen problemas familiares o incluso que padezcan de algún tipo de marginación cultura o educativa. Finalmente, nos atrevemos a decir que no podemos hablar de violencia escolar, sino entendemos la violencia estructural que se reproduce en la sociedad.

\section{REFERENCIAS}

Acosta, C. (2015). La violencia y victimización verbal, factores que impactan en los alumnos. Caso: COBACH-EMSAD 18 "Chiná". Investigación. Revista electrónica multidisciplinaria de investigación y docencia. Recuperado de: http://instcamp.edu.mx/wpcontent/uploads/2015/12/A\%C3\%B1o2015No7.pdf

Albores-Gallo, L., Sauceda-García, Ruiz, J. M. y Roque-Santiago, E. (2011). El acoso escolar (bullying) y su asociación con trastornos psiquiátricos en una muestra de escolares en México. Salud pública de México, 53(3): pp. 220-227. 
Armero, P., Bernardino, B. y Bonet, C. (2011). Acoso escolar. Pediatría de Atención Primaria, 23 (52): pp. 661-670.

Ayala-Carrillo, M. (2015). Violencia escolar: un problema complejo. Ra Ximhai, 11 (4): pp. 493-509.

Bertely, M. (2000). Conociendo nuestras escuelas: un acercamiento etnográfico a la cultura escolar. México, Paidós.

Bertely, M. (2000). Conociendo nuestras escuelas. Un acercamiento etnográfico a la cultura escolar. Barcelona: Paidós.

Blanchard, M. (2007). Acoso escolar. Desarrollo, prevención y herramientas de trabajo. Madrid: Ediciones Narcea.

Carney A. G. $\quad$ G Merrell K. (2001). Bullying in schools: Perspectives on understanding and preventing an international problem. School Psychology Internatio nal, 22 (3): pp. 364-382.

Collino, M. (2017). La Educación Emocional en el Aula. Argentina: Universidad Siglo 21.

Escudero, J.M. (2005). Fracaso escolar, exclusión educativa: ¿de qué se excluye y cómo?. Profesorado, Revista de currículum y formación del profesorado, 1, (1): pp. 125.

Fernanda, M., Villota, E. y Garzón, F. (2015). El acoso escolar. Saber ciencia y libertad, 10 (1): pp. 219-233.

Fernández, I., Villaoslada, E. y Funes, S. (2002). Conflicto en el centro escolar. Madrid. Catarata

Fuller, N. (2001). No uno sino muchos rostros. Identidad masculina en el Perú urbano. En Mara Viveros, José Olavarría y Norma Fuller, Hombres e identidades de género (pp. 265-270). Bogotá: Universidad Nacional de Colombia.

Gairín, J., Armengol, C. y García, S. El «bullying» escolar. Consideraciones organizativas y estrategias para la intervención. Educación XX1, 16 (1): pp. 19-38. 
Garbanzo, M. (2007). Factores asociados al rendimiento académico en estudiantes universitarios, una reflexión desde la calidad de la educación superior pública. Educación, 31 (1): pp. 43 63.

García, A. y Ferreira, G. (2005). La convivencia escolar en las aulas. International Journal of Developmental and Educational Psychology, 2 (1): pp. 163-183.

García, M. y Ascensio, C. (2015). Bullying y violencia escolar: diferencias, similitudes, actores, consecuencias y origen. Revista Intercontinental de Psicología y Educación, 17 (2): pp. 938.

Geertz, C. (1986). La interpretación de las culturas. Barcelona Gedisa SA.

Gómez, M. C. y da Resurrección, A. (2017). Intervention Strategies in disruptive behavior. Educação or Escrito, Porto Alegre, 8 (2): pp. 278-293.

Guber, R. (2001). La etnografía. Método, campo y reflexividad. Bogotá: Editorial Norma.

Hamodi, C. y Jiménez, L (2018), Modelos de prevención del bullying: ¿qué se puede hacer en educación infantil? Revista de Investigación Educativa de la REDIECH, 9 (16): pp. 2950.

Jankue, K. (2016). Prevención e intervención escolar ante actos autolíticos (trabajo final de grado no publicado). España: Universitat Jaume I.

Jociles, M. (1999). Las técnicas de investigación en antropología. Recuperado de: http://www.ugr.es/ pwlac/G15_01MariaIsabel_Jociles_Rubio.pdf

Loredo, A., Perea, A. y López, G. (2008). “Bullying”: acoso escolar. La violencia entre iguales. Problemática real en adolescentes. Acta Pediátrica de México, 29 (4): pp. 210-214.

Maquilón, J. (2011). La formación del profesorado en el siglo XXI Propuestas ante los cambios económicos, sociales y culturales. España: Universidad de Murcia.

Mena, L., Fernández, M. y Riviére, J. (2010). Desenganchados de la educación: procesos, experiencias, motivaciones y estrategias del abandono y del fracaso escolar. Revista de Educación, número extraordinario, 1: pp. 119-145 
Moreno, J., Cervelló, E., Martínez, C. y Villodre, N. (2007). Los comportamientos de disciplina e indisciplina en educación física. Revista Iberoamericana de Educación, 44: pp. 167-190.

Muñoz, G. (2008). Violencia escolar en México y en otros países. Comparaciones a partir de los resultados del Instituto Nacional para la Evaluación de la Educación. Revista Mexicana de Investigación Educativa, 13 (39): pp. 1195- 1228.

Orrego, J. (2014). Tesis doctoral. Perfil psicosocial y modelo de intervención en adolescentes con problemas graves del comportamiento. España: Universidad Oviedo.

Ovalles, A. (2017). Tesis Doctoral. Estilos educativos familiares y conductas disruptivas en el adolescente. Aplicaciones educativas. España: Universidad Complutense de Madrid.

Pérez, A. (2012). La etnografía como método integrativo. Revista Colombiana de Psiquiatría, 41 (2): pp. 421-428.

Rockwell, E. (1995). La escuela cotidiana. Ciudad de México: Fondo de Cultura Económica.

Rodríguez, A. y Mejía, Y. (2012). Bullying: un fenómeno por transformar. Duazary, 9 (1): pp. 98104.

Rueda, M. y Campos, M. A. (1992). Investigación etnográfica en educación. México: Ciudad de México.

Sánchez, J. M., Hernández, C., Villamar, B. A., Rodríguez, D. B., Lira, G. C., Ruiz, A. y Diego, C. M. (2013). ¿Dónde está la violencia escolar?. En F. J. Pedroza y S. J. Aguilera (Eds.), La construcción de identidades agresoras: el acoso escolar en México (pp. 15-30). México: CONACULTA

Santoyo, D. y Frías, S. (2014). Acoso escolar en México: actores involucrados y sus características. Revista Latinoamericana de Estudios Educativos (México), vol. 44 (4): pp. $13-41$.

Sautu, R., Boniolo, P., Dalle, P. y Elbert, R. (2005). Manual de metodología: construcción del marco teórico, formulación de los objetivos y elección de la metodología. Argentina: CLACSO.

Tresgallo, E. (2008). Violencia escolar (“Bullying”): documento para padres y educadores. Revista Española de Orientación y Psicopedagogía, 19 (3): 328-333. 
UNESCO, (2013). Análisis del clima escolar: ¿Poderoso factor que explica el aprendizaje en América Latina y el Caribe?. España: Santillana.

Vásquez, F. (2010). Estrategias de enseñanza. Investigaciones sobre didáctica en instituciones educativas de la ciudad de Pasto. Bogotá: Universidad de la Salle.

Vega, J. (2018). Prevalencia del bullying en México: un meta-análisis del bullying tradicional y cyberbullying. Diversitas: Perspectivas en Psicología, 15 (1): pp. 113-129.

Vega, M., González, G., Valle, M., Flores, M., Vega, A. (2013). Acoso escolar en la zona metropolitana de Guadalajara, México: prevalencia y factores asociados. Salud Colectiva, 9 (2): pp. 183-194

Conflicto de intereses / Competing interests:

Los autores declaran que no incurren en conflictos de intereses.

Rol de los autores / Authors Roles:

Yareli Reyes: conceptualización, curación de datos, análisis formal, adquisición de fondos, investigación, metodología, administración del proyecto, recursos, software, supervisión, validación, visualización, escritura preparación del borrador original, escritura - revisar \& amp; edición.

John Acuña: conceptualización, investigación, metodología, administración del proyecto, recursos, software, supervisión, validación, visualización, escritura - preparación del borrador original, escritura - revisar \& amp; edición.

Fuentes de financiamiento / Funding:

Los autores declaran que no recibieron un fondo específico para esta investigación.

Aspectos éticos / legales; Ethics / legals:

Los autores declaran no haber incurrido en aspectos antiéticos, ni haber omitido aspectos legales en la realización de la investigación. 